\title{
CASOS VERIFICADOS DE USO ILEGIITIMO DE SOFTWARE DE VIGILANCIA POR PARTE DE GOBIERNOS DE AMÉRICA LATINA 2015-2016
}

\section{VERIFIED CASES OF UNLAWFUL USE OF SURVEILLANCE SOFTWARE BY LATIN AMERICAN GOVERNMENTS 2015-2016}

\author{
RAFAEL BONIFAZ ${ }^{1}$ \\ ANDRÉS DELGADO-RON ${ }^{2}$
}

\footnotetext{
${ }^{1}$ Universidad de Buenos Aires, Buenos Aires, Argentina (rafael.bonifaz@posgrado.economicas.uba.ar).

${ }^{2}$ Docente Investigador Universidad Tecnológica Equinoccial, Quito, Ecuador (andres.delgadoron@ute.edu.ec).
} 
inlllis 


\section{CASOS VERIFICADOS DE USO \\ ILEGIITIMO DE SOFTWARE DE VIGILANCIA POR PARTE DE GOBIERNOS DE AMÉRICA LATINA 2015-2016}

\section{VERIFIED CASES OF UNLAWFUL USE OF SURVEILLANCE SOFTWARE BY LATIN AMERICAN GOVERNMENTS 2015-2016}

Rafael Bonifaz, Andrés Delgado-Ron

Palabras clave: seguridad informática, vigilancia, espionaje, América Latina, hacking

Key words: information security, surveillance, espionage, Latin America, hacking

\section{RESUMEN}

El presente artículo es una investigación bibliográfica y análisis exploratorio acerca de la vigilancia estatal en internet en América Latina. Describe los alcances de las capacidades de vigilancia gubernamental mediante el estudio de la documentación publicada por Edward
Snowden y Wikileaks entre 2011 y 2017. Resume la presencia de la industria de vigilancia en América Latina y presenta cuatro casos de estudio sobre uso ilegítimo de software de espionaje y vigilancia en la región. 


\section{SUMMARY}

The present article is a bibliographical investigation and exploratory analysis about State-sponsored online surveillance in Latin America. It describes public surveillance capabilities by studying the documentation published by Edward Snowden and Wikileaks from
2011 to 2017. In addition, the article summarizes the known comercial transactions related to the surveillance industry in Latin America. Finally, it presents four case-studies on the illegitimate use of surveillance and espionage software in the region.

\section{INTRODUCCIÓN}

El presente trabajo es una investigación bibliográfica y análisis exploratorio de la documentación publicada sobre la vigilancia estatal en Internet en América Latina. El objetivo es identificar casos de espionaje ilegítimo por parte de sus gobiernos durante los años 2015 y 2016. El artículo está dividido en tres secciones. La primera introduce al lector en las capacidades actuales de vigilancia gubernamental a partir del estudio de documentos publicados durante la última década, con un enfoque especial en las capacidades del gobierno de Estados Unidos, por representar el estado del arte en cuanto a capacidades tecnológicas de espionaje. La segunda sección analiza el uso ilegítimo de software de infiltración y vigilancia por parte de los gobiernos de América Latina. Finalmente, en la última sección se presentan las conclusiones del artículo.

\section{VIGILANCIA GLOBAL}

En junio de 2013, Edward Snowden, exanalista de la Agencia Nacional de Seguridad de los Estados Unidos (NSA por sus siglas en inglés), filtró miles de documentos clasificados a los periodistas Glenn Greenwald, Ewen MacAskill y Laura Poitras. Los documentos contie- nen herramientas de entrenamiento a oficiales de seguridad, comunicaciones internas, información sobre tratados entre agencias de inteligencia, y otro tipo de documentos que, en forma conjunta, muestran las capacidades de vigilancia del gobierno de Estados Unidos así como 
la capacidad tecnológica existente, en general (Greenwald, 2014). Mediante el programa SIGAD US-984XN —-mejor conocido como PRISM-, por ejemplo, la NSA obtenía información de los usuarios de Apple, Facebook, Google, Microsoft, Skype, Yahoo y Youtube. La información recopilada es aquella normalmente utilizada en los servicios de dichas empresas: correo electrónico, chat, imágenes, videoconferencias, llamadas de voz, etcétera (Gellman \& Poitras, 2013).

Bonifaz (2017) detalla las distintas formas en que la NSA recolecta información y los sistemas que tiene para analizarla, las cuáles también están codificadas bajo diferentes códigos que se explicarán a continuación. La leyenda "3rd Party/Liaison", por ejemplo, hace referencia a la recolección que la NSA realiza en alianza con otros países; "Regional" se refiere a la interceptación de comunicaciones de telefonía celular que se realiza en embajadas a nivel global; "CNE" se refiere a la interceptación que se realiza mediante ataques informáticos; finalmente, "Large Cable" es la interceptación que se realiza en conjunto a las empresas norteamericanas que administran los cables de fibra óptica como AT\&T y Verizon. La llustración 1 muestra las formas de recolección de información de la NSA a nivel mundial.

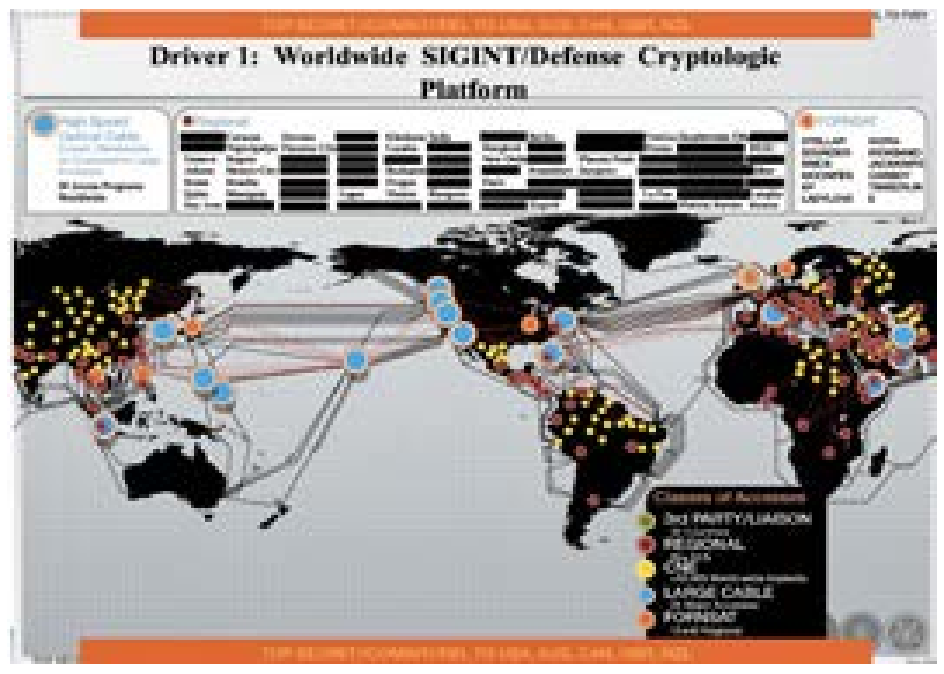

llustración 1: Formas en la que la NSA recolecta información. Fuente: ("Worldwide SIGINT/Defense Cryptologic Platform", s/f) 
La información recolectada por estos sistemas era procesada por diversos sistemas para su posterior análisis. La bases de datos, interfaces y programas que integraban "Xkeyscore" funcionaban de manera similar a un motor de búsqueda en Internet (Ali \& Hudaib, 2014). Para el año 2009, Xkeyscore tenía la capacidad analizar todo el tráfico de internet capturado por un tiempo de 3 a 5 días, en el caso del contenido, y de 30 a 45 días, en el caso de los metadatos (Lee, Greenwald, \& Marquis-Boire, 2015).

Por su mandato, la NSA es la agencia encargada de recopilar y analizar comunicaciones extranjeras, incluido internet, para defensa de la seguridad nacional. Además, la Agencia Central de Inteligencia (CIA por sus siglas en inglés) es la encargada de recopilar y analizar información de individuos y gobiernos extranjeros. El 7 de marzo de 2017, Wikileaks empezó a publicar la filtración conocida como VAULT7 donde se muestran las herramientas desarrolladas por la CIA para realizar ataques informáticos. Los ataques incluyen a los sistemas operativos Windows, MacOS, Linux, Android e iOS; así como también software para infectar ruteadores, televisores Samsung, autos, entre otros (Wikileaks, 2017).

El 14 de abril de 2017, un grupo anónimo conocido como The Shadow
Brokers, publicó el código fuente de software malicioso desarrollado por la NSA conocido como EternalBlue. Este fue utilizado poco tiempo después en WannaCry y Petya, ransonwares 1 que afectaron a sistemas informáticos a nivel mundial en ese año (Barret, 2017).

Tanto la CIA como la NSA hacen uso de vulnerabilidades de cero días. Es decir, fallas de seguridad que tienen los sistemas informáticos que son conocidas únicamente por el atacante e ignoradas por usuarios y desarrolladores. Existe evidencia que estas agencias ocasionalmente prefieren no reportar las fallas de seguridad a los desarrolladores de los sistemas de software - lo que los haría más seguros. En su lugar, utilizan esta información con el fin de explotarlas. De esta manera, incrementan el riesgo de que los usuarios sean víctimas de un ataque informático (Fidler, 2014).

Existen al menos 160 empresas que venden software de vigilancia a nivel mundial (Wikileaks, 2011b, 2014). Aunque la mayoría operan en secreto, varias filtraciones de información han permitido entender sus capacidades y forma de operar. Hacking Team y Finfisher son empresas que ofertan y venden software de espionaje para tomar control de forma remota de una computadora o un teléfono celular, convirtiéndolos

\footnotetext{
${ }^{1}$ Ransomware es un software malicioso que cifra información. Usualmente quienes lo usan piden dinero a cambio de la clave para descifrarla.
} 
en dispositivos de seguimiento. Previo a la revelación de sus operaciones, este software tenía la capacidad de encender de forma remota la cámara y el micrófono para poder espiar el dueño del dispositivo. Toda la información dentro del dispositivo podía ser accedida por el analista de inteligencia. Conversaciones de chat, historiales de navegación, ubicación geográfica, entre otras, forma parte de la información que podía ser extraída con el software desarrollado por estas empresas (W. R. Marczak, Scott-Railton, Marquis-Boire, \& Paxson, 2014).

Además empresas como VASTech venden software para interceptar de manera masiva comunicaciones telefónicas. Según un documento de publicidad de la solución Zebra, VASTech afirma tener la capacidad de interceptar y almacenar contenido y metadatos de las comunicaciones. Un solo equipo Zebra tiene la capacidad de interceptar 100.000 llamadas de manera simultánea. Doscientos equipos Zebra pueden trabajar de forma conjunta (Wikileaks, 2011a). Es decir, para 2011 con la solución de VASTech un gobierno podría interceptar hasta veinte millones de llamadas simultáneamente.

El acelerado avance de la tecnología ha incrementado también de forma exponencial las capacidades de recolección y almacenamiento de datos, reduciendo drásticamente el costo de la inteligencia de señales. Esta sección ha demostrado el alcance que puede llegar a tener un sistema integrado de vigilancia. Si bien es cierto que los países del sur global aún no cuentan con este tipo de infraestructura de espionaje, tampoco se debe olvidar que los ciclos de adopción de tecnología son cada vez más cortos y los precios cada vez más asequibles.

\section{ESPIONAJE EN AMÉRICA LATINA}

Investigaciones previas han señalado la dificultad de aproximarse al estudio de la vigilancia en América Latina. Los regímenes de vigilancia responden a motivaciones distintas a aquellas de países industrializados y, en la región, han sido impulsados en gran medida por la inseguridad generalizada. Gobiernos, criminales y sociedad civil han implementado mecanismos de vigilancia principalmente como medida de protección ante grupos adversos (Botello, 2015). Años atrás, los países de bajos y medianos ingresos veían sus capacidades de espionaje e inteligencia limitadas por los altos costos que estas suponen. Sin embargo, los avances tecnológicos de países desarrollados han facilitado también el espionaje en países 
periféricos. Según Albirini (2008), "el gobierno de los Estados Unidos ha promovido leyes que obligan a todos los fabricantes de tecnología de comunicación a desarrollar capacidades de vigilancia y al mismo tiempo a limitar el hardware y el software que proporcionan cifrado". Asimismo, otros países desarrollados han impuestos estándares de vigilancia que, finalmente, se han transferido también al sur global. Adicionalmente, la alta demanda de software de vigilancia generada por países ricos ha impulsado el crecimiento de un mercado mundial de vigilancia (Ablon, Libicki, \& Golay, 2014) que ha sabido encontrar clientes entre países que apenas están desarrollando un marco regulatorio frente a estas tecnologías.
En agosto de 2016, Transparency Toolkit y Privacy International presentaron el Índice de la Industria de Vigilancia (Cox, 2016), el cual contiene más de 520 empresas a nivel mundial. Esta base de datos recopila información pública disponible sobre firmas que venden software de vigilancia a gobiernos alrededor del mundo. En la Tabla 1 se presentan las empresas que operan en América Latina, así como la tecnología que se ha provisto durante los últimos años. Es importante señalar que los datos presentados corresponden a registros de transacciones comerciales y no es posible determinar el estado activo de ninguna de estas tecnologías.

\begin{tabular}{|c|c|c|}
\hline País & Proveedor(es) & Tecnología adquirida (\#) \\
\hline Argentina & Blue Coat, Otro/Desconocido & Inspección profunda de paquetes (1) \\
\hline Bolivia & Blue Coat & Inspección profunda de paquetes (1) \\
\hline Brasil & Blue Coat, EXFO, Hacking Team, Otro/Desconocido & $\begin{array}{l}\text { Intercepción aérea (4) } \\
\text { Software de intrusión (1) } \\
\text { Inspección profunda de paquetes (1) }\end{array}$ \\
\hline Chile & Blue Coat, Hacking Team, Otro/Desconocido & $\begin{array}{l}\text { Intercepción aérea (2) } \\
\text { Software de intrusión (1) } \\
\text { Inspección profunda de paquetes (1) }\end{array}$ \\
\hline Colombia & $\begin{array}{l}\text { Blue Coat, Digivox, EXF0, Hacking Team, Network Critical, NICE } \\
\text { Systems, Oakley Networks, Smith Myers, Spectra, Tracespan, } \\
\text { Verint, Otro/Desconocido }\end{array}$ & $\begin{array}{l}\text { Intercepción aérea (5) } \\
\text { Pinchamiento de fibra (5) } \\
\text { Infraestructura de red y servicios (2) } \\
\text { Centro de monitoreo (1) } \\
\text { Interceptación legal (1) } \\
\text { Software de intrusión (1) } \\
\text { Inspección profunda de paquetes (1) }\end{array}$ \\
\hline
\end{tabular}




\begin{tabular}{|c|c|c|}
\hline Costa Rica & Blue Coat & Inspección profunda de paquetes (1) \\
\hline Ecuador & Blue Coat, Hacking Team & $\begin{array}{l}\text { Software de intrusión (1) } \\
\text { Inspección profunda de paquetes (1) }\end{array}$ \\
\hline El Salvador & Otro/Desconocido & Intercepción aérea (1) \\
\hline Guatemala & Blue Coat & Inspección profunda de paquetes (1) \\
\hline Honduras & Hacking Team & Software de intrusión (1) \\
\hline Jamaica & Blue Coat & Inspección profunda de paquetes (1) \\
\hline México & $\begin{array}{l}\text { Blue Coat, EXF0, FinFisher, Gamma International, Hacking Team, } \\
\text { NSO, Speech Technology Center, Verint }\end{array}$ & $\begin{array}{l}\text { Software de intrusión (13) } \\
\text { Intercepción aérea (9) } \\
\text { Inspección profunda de paquetes (2) } \\
\text { Infraestructura de red y servicios (1) } \\
\text { Centro de Monitoreo (1) } \\
\text { Interceptación legal (1) } \\
\text { Biometría (1) }\end{array}$ \\
\hline Nicaragua & Blue Coat & Inspección profunda de paquetes (1) \\
\hline Panamá & $\begin{array}{l}\text { Blue Coat, EXF0, FinFisher, Gamma International, Hacking Team, } \\
\text { NSO }\end{array}$ & $\begin{array}{l}\text { Software de intrusión (3) } \\
\text { Inspección profunda de paquetes (1) }\end{array}$ \\
\hline Paraguay & Gamma International, FinFisher, Otro/Desconocido & $\begin{array}{l}\text { Intercepción aérea (3) } \\
\text { Software de intrusión (1) }\end{array}$ \\
\hline Perú & Blue Coat & Inspección profunda de paquetes (1) \\
\hline $\begin{array}{l}\text { República } \\
\text { Dominicana }\end{array}$ & Blue Coat & Inspección profunda de paquetes (1) \\
\hline Venezuela & Blue Coat, FinFisher, Gamma International & $\begin{array}{l}\text { Intercepción aérea (1) } \\
\text { Software de intrusión (1) } \\
\text { Inspección profunda de paquetes (1) }\end{array}$ \\
\hline
\end{tabular}

Tabla 1: Empresas distribuidoras de software de vigilancia que registran transacciones comerciales en América Latina. Fuente: Transparency Toolkit \& Privacy Internatioanl < https://sii.transparencytoolkit.org/>. Elaboración: Autores.

Debido al secretismo con que de cerca con el fin de entender los fines operan las agencias de seguridad e inteli- $\quad$ y alcances reales de la vigilancia gubergencia, es difícil realizar estudios exhaus- namental en América Latina. Los autores tivos sobre sus prácticas. Sin embargo, hemos seleccionado cuatro casos de eslas eventuales filtraciones públicas sobre tudio para realizar una aproximación al sus operaciones deben ser examinadas tema en la región. 


\section{HACKING TEAM PRESENTE EN 12 PAÍSES}

En julio de 2015, se hicieron públicos 400 gigabytes de información de la empresa italiana Hacking Team. La seguridad informática de la empresa fue vulnerada: correos electrónicos, archivos digitales, código fuente, y otros, fueron expuestos en Internet. Según un informe realizado por la organización Chilena Derechos Digitales, los documentos muestran que Brasil, Chile, Colombia, Ecuador, Honduras, México y Panamá compraron licencias de software de esta empresa; mientras que Argentina, Guatemala, Paraguay, Uruguay y Venezuela mantuvieron negociaciones que, hasta julio de 2015, no se concretaron. Los países que compraron el software de Hacking Team tienen legislación que limita el uso de este tipo de herramientas. En todos los países citados en dicho informe, se necesita la orden de un juez y la presunción de haber cometido un delito para poder interceptar comunicaciones. La autora de la investigación destaca que "la naturaleza del software de Hacking Team es tan invasiva que tiene acceso a todo, incluyendo a las comunicaciones especialmente protegidas, sin que existan mecanismos adecuados para controlarIo" (Pérez de Acha, 2016, p. 29).

Galileo, el producto de Hacking Team, es un software malicioso que al infectar una computadora o teléfono móvil permite tomar control del mismo de manera remota. Se podría encender el micrófono o la cámara de un dispositivo, acceder a la geolocalización, leer las conversaciones de chat y correos electrónicos almacenados en el dispositivo, etcétera. Las agencias de seguridad podrían acceder a toda la información que se almacena en el teléfono celular de manera remota.

Este tipo de software tiene usos legítimos como la lucha contra el crimen organizado. Sin embargo, el informe de Derechos Digitales, respaldado por los correos de Hacking Team, muestra que en el caso de Ecuador, México y Panamá se utilizó este software para espionaje político.

En el caso del expresidente de Panamá existe una orden de prisión por "interceptar las comunicaciones de unas 150 personas entre empresarios, periodistas, dirigentes de la sociedad civil y políticos opositores a su gobierno" (Agencia AFP, 2015). Según un análisis realizado por un miembro del proyecto Tor, la Secretaría Nacional de Inteligencia de Ecuador (SENAIN) utilizó el software para espionaje político. Se destaca el caso realizado a Hacking Team para espiar a jueces, al Consejo Nacional Electoral, a la Confederación de Nacionalidades Indígenas del Ecuador, al mo- 
vimiento político Pachakutik, entre otros (Ilv, 2015). El programa Visión 360 de la televisión ecuatoriana habló de la posibilidad de haber utilizado el software de Hacking Team para espiar a miembros del grupo de activismo ecológico "Yasunidos" y al activista político Carlos Vera (Tinoco, 2015).
Chile y México se encuentran entre los cinco países que más dinero gastaron en productos de Hacking Team (Arturo, 2015). En la ilustración 2 se pueden apreciar los gastos de cada país en software de espionaje distribuido por Hacking Team.

\section{HACKING TEAM EN AMÉRICA LATINA}

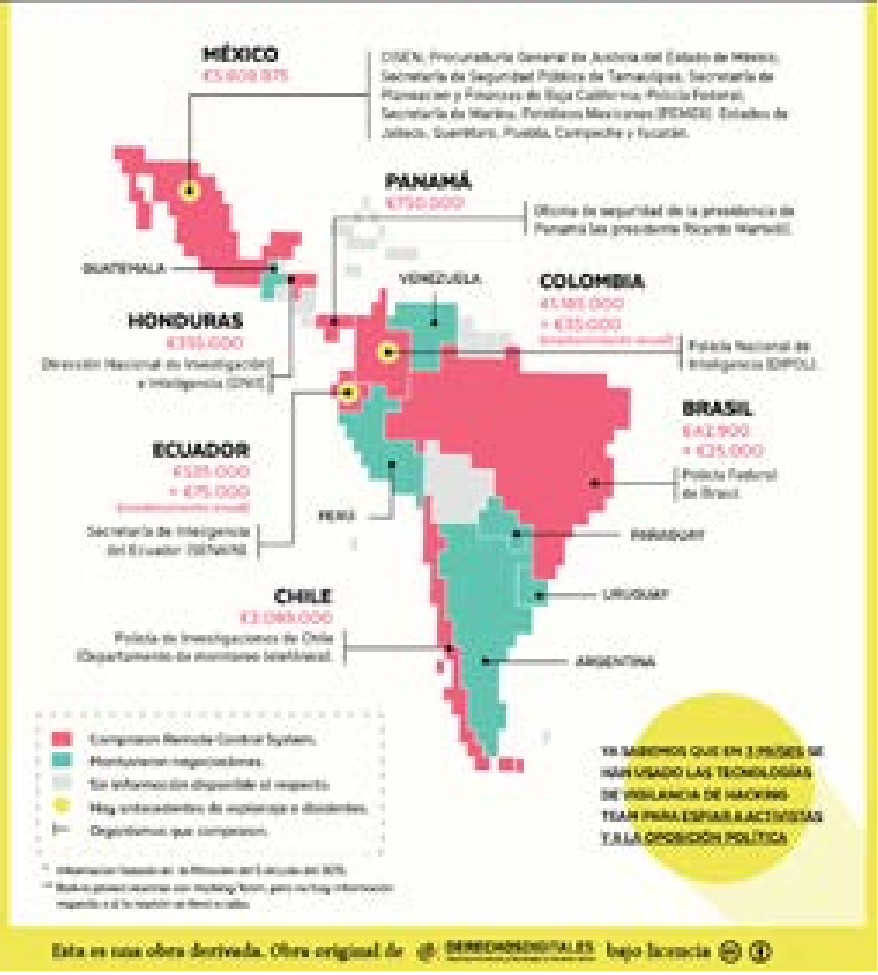

Ilustración 2: Transacciones comerciales de Hacking Team en América Latina. Fuente: Derechos Digitales. Adaptación: Autores. 


\section{FINFISHER EN MÉXICO, VENEZUELA Y PARAGUAY}

En octubre de 2015, el laboratorio de investigación The Citizen Lab de la Universidad de Toronto realizó una investigación sobre el software de vigilancia de la empresa Fin Fisher. Esta empresa vende software espía similar al de Hacking Team que, además de otras funcionalidades, permite tomar control remoto de un dispositivo electrónico (B. Marczak, Scott-Railton, Senft, Poetranto, \& McKune, 2015).

La investigación consistió en escanear todas las direcciones IPV4 de Internet utilizando las herramientas de Zmap (The ZMap Team, 2017). Al realizar el escaneo se hizo cierto tipo de consultas a los servidores y, según las respuestas, se pudo identificar que servidores utilizaban software de Fin Fisher. En particular, se identificaron dos tipos de ser- vidores. Los que reciben las comunicaciones de un objetivo/víctima vigilado y los servidores relés que intentan ocultar esas comunicaciones.

Cuando el dispositivo de un blanco de vigilancia es infectado por el software de Fin Fisher, el equipo intentará conectarse a un servidor remoto conocido como FinSpy Master. Este servidor tiene la funcionalidad de tomar control remoto de los equipos infectados. Para ocultar que el equipo infectado está conectándose a un servidor de una organización estatal se utilizan los servidores relés de FinSpy. De esta manera, en lugar de ver conexiones a la policía nacional, por ejemplo, se verán conexiones a un servidor en algún país remoto. En la ilustración 3 se puede ver la descripción completa.

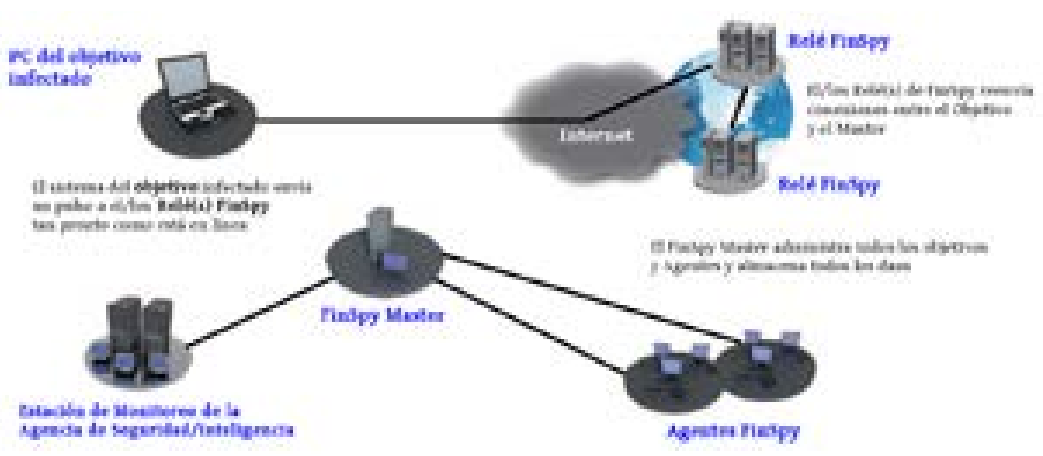

Ilustración 3: Monitoreo remoto y Soluciones de Infección de FinSpy. Fuente: Wikileaks ("Remote Monitoring \& Infection Solutions: FINSPY", s/f) 
El estudio identificó 33 posibles clientes ubicados en 32 países. Se identificó que México, Venezuela y Paraguay son clientes del software de FinFisher. En el caso de México y Venezuela, ya se conocía del uso de esta herramienta. En el caso de Paraguay, la investigación reveló por primera vez el uso de este software en dicho país. Debido a las limitaciones propias del estudio, el reporte no detalla que organizaciones operan el software en ninguno de estos tres países ni contra quienes dirigen sus operaciones.

\section{PACKRAT EN ARGENTINA, BRASIL, ECUADOR \& VENEZUELA}

A fines de 2015, investigadores de The Citizen Lab develaron una campaña activa de desinformación, phishing y malware operando en Argentina, Brasil, Ecuador y Venezuela. Las víctimas eran principalmente opositores políticos de los regímenes de turno así como periodistas independientes. El informe concluye que es muy probable que los atacantes estén auspiciados por uno o varios gobiernos dada su falta de preocupación por ser descubiertos, su persistencia y sus objetivos (Scott-Railton, Marquis-Boire, Guarnieri, \& Marschalek, 2015). La denominación del atacante se da precisamente por su preferencia por el uso de Paquetes de Troyanos de Acceso Remoto (Packed Remote Access Trojan). CyberGate y AlienSpy son algunos de los implantes utilizados para infectar los equipos.

Las características del funcionamiento de estos códigos maliciosos son similares a las de Hacking Team y FinFi- sher. Permite tomar control del sistema infectado y reportar la actividad que sucede en el mismo. De esta manera, se podría activar de forma remota la cámara y el micrófono del equipo, buscar contraseñas almacenadas, capturar la captura de teclas para robar contraseñas, capturas, de pantalla, etcétera.

Existían varias formas de infectar los dispositivos de los sujetos objetivos de vigilancia. Una de ellas es a través de correo electrónico mediante un archivo adjunto infectado. También era posible utilizar enlaces a una página web maliciosa. En el primer caso, era necesario abrir el archivo en una computadora mientras que en el segundo la infección sucedía desde la página web.

En el caso de Ecuador, los correos electrónicos estudiados demuestran intentos de ataque contra opositores al gobierno del expresidente Rafael Correa. Uno de ellos, está dirigido a Martha Roldós y proviene de un supuesto "Mo- 
vimiento anticorreista". El mismo incluye un documento de Word con un código malicioso para permitir el control remoto de la computadora. El informe recalca el uso de ingeniería social focalizado en los adversarios al gobierno.

Otra técnica fue el uso de dominios falsos para simular sitios legítimos. Por ejemplo se utilizaba el dominio "ecuadorenvivo.co" para simular el sitio web legítimo "ecuadorenvivo.com". Tras ingresar al sitio falso, a la víctima se le pedía descargar un complemento para el navegador. El complemento a descargar sería software malicioso para infectar el equipo de la víctima.
En el caso de Argentina el estudio afirma que el fiscal Alberto Nissman, asesinado a inicios de enero de 2015, fue espiado utilizando este tipo de ataque. Lo mismo se dice del periodista Jorge Lanata.

Según la investigación, todos los ataques se realizados en Venezuela, Brasil, Ecuador y Argentina están relacionados entre sí y utilizan infraestructura compartida. El informe presume que estos ataques tienen que ver con uno o varios Estados, aunque no descarta por completo la posibilidad de actores no gubernamentales.

\section{NSO Y EL CASO GOBIERNO ESPÍA EN MÉXICO}

En agosto de 2016, investigadores de la Universidad de Toronto realizaron una investigación sobre el espionaje realizado al activista Ahmed Mansoor en los Emiratos Arabes Unidos mediante Pegasus, un software malicioso de la empresa NSO Group. Este software tiene funcionalidades similares a los programas presentados en las secciones anteriores. Es decir, permite tomar control de forma remota de equipos informáticos. La investigación determinó cuáles eran los países que más utilizan este software. Al igual que en el caso de Hacking Team, la lista también es encabezada por México
(Scott-Railton \& Marczak, 2016).

Motivados por este informe, las organizaciones Artículo 19 (oficina para México y Centroamérica), la Red de Defensa de Derechos Digitales (R3D) y SocialTIC realizaron en conjunto el reporte "Gobierno Espía" sobre el caso NSO. En el mismo, se evidencia que el software de vigilancia fue utilizado para atacar a periodistas y activistas que denunciaron casos de corrupción en México (Article 19, R3D, \& SocialTIC, 2017). Los intentos de infección se realizaron a través de mensajes SMS. Las víctimas incluyen al Centro de Derechos Humanos Miguel 
Agustín Pro Juárez, que denunció el caso de los 43 estudiantes desaparecidos en Ayotzinapa; Aristegui Noticias y Mexicanos contra la Corrupción y la Impunidad, activos en el caso conocido como Casa Blanca y otros que involucran al presidente mexicano Enrique Peña Nieto;
Carlos Loret de Mola, quien reportó sobre el enfrentamiento de Tanhuato donde murieron 42 civiles, entre otros. En el caso de Carmen Aristegui se intentó infectar el teléfono de su hijo, que en ese entonces era menor de edad.

\section{CONCLUSIONES}

Los casos presentados en este estudio demuestran la existencia y proliferación del espionaje gubernamental en América Latina, esto a pesar que la mayoría de países de la región cuenta con algún tipo de legislación que defiende la privacidad, usualmente a nivel constitucional (Caldera \& Parraguez Kobek, 2016). En el caso de Estados Unidos, agencias como la CIA y la NSA cuentan con gran capacidad de tecnología para crear sus propias herramientas. En el caso de los países de América Latina, de momento no se ha demostrado que exista capacidad de desarrollo de software de espionaje en la región mas es evidente la presencia de un mercado de vigilancia que satisface estas necesidades.

La fortaleza de nuestro estudio radica en la sistematización de documentación pública sobre casos documentados de espionaje ilegítimo por parte de gobiernos. Sin embargo, el secretismo con el que las agencias de inteligencia y seguridad operan dificulta una comprensión exhaustiva de sus acciones. La legitimidad y responsabilidad en las acciones de espionaje emprendidas por un gobierno solo pueden ser evaluadas con información fidedigna y no deberían depender de eventuales filtraciones de información (Setty, 2015). Los países de la región deben desarrollar políticas que permitan una mayor transparencia y control de sus agencias de inteligencia y seguridad así como una mayor fiscalización a las compras públicas de software espía.

Actualmente, muchos países de América Latina están desarrollando estrategias de ciberdefensa o ciberseguridad, a menudo centradas exclusivamente en asuntos militares o de inteligencia. Sin embargo, otros elementos son necesarios para lograr el balance adecuado entre la seguridad y los derechos humanos, tales como la privacidad y la libertad de expresión y asociación. La colabo- 
ración multistakeholder para la gober- de poder. Asimismo, la ciudadanía debe nanza de internet podría generar mayor estar informada sobre los riesgos a la primadurez en materia de ciberseguridad vacidad debido a la vigilancia masiva. Por (Maciel, Foditsch, Belli, \& Castellon, n.d.). lo que es importante que los temas relaEs importante mejorar los controles so- cionados con vigilancia se abran al debabre las agencias de inteligencia de cada te público y sus riesgos sean entendidos uno de los países para evitar los abusos por los ciudadanos. 


\section{BIBLIOGRAFÍA}

Ablon, L., Libicki, M. C., \& Golay, A. a. (2014). Markets for Cybercrime Tools and Stolen Data. National Security Research Division, 1-85. https://doi.org/10.7249/j.ctt6wq7z6

Agencia AFP. (2015, December 22). Corte Suprema de Panamá ordena detención de expresidente Martinelli. Diario El Heraldo. Retrieved from http://www.elheraldo.hn/ mundo/913506-466/corte-suprema-de-panamá-ordena-detención-de-expresidente-martinelli

Albirini, A. (2008). The Internet in developing countries: a medium of economic, cultural and political domination. International Journal of Education and Development Using Information and Communication Technology (IJEDICT), 4(1), 49-65. Retrieved from http://ijedict.dec. uwi.edu//viewarticle.php?id=360 Ali, A., \& Hudaib, Z. (2014). Comprehensive Social Media Security Analysis \& XKeyscore Espionage Technology, (8), 97-158.

Article 19, R3D, \& SocialTIC. (2017). Gobierno Espía: Vigilancia sistemática a periodistas y defensores de derechos humanos en México. Retrieved from https://r3d.mx/ gobiernoespia
Arturo, Á. (2015). México, el principal cliente de una empresa que vende software para espiar. Animal Político. Animal Político. Retrieved from http://www. animalpolitico.com/2015/07/ empresa-de-hackers-exhibida-por-venta-de-software-espia-a-paises-represores-y-mexico-resulta-su-principal-cliente/

Barret, B. (2017). The Encryption Debate Should End Right Now. WIRED. Retrieved from https://www.wired.com/story/ encryption-backdoors-shadow-brokers-vault-7-wannacry/

Bonifaz, R. (2017). The NSA Surveillance Capabilities According to the Snowden Documents. In Actas del IX Congreso Iberoamericano de Seguridad Informática CIBSI2017. Buenos Aires: Facultad de Ingeniería, Universidad de Buenos Aires.

Botello, N. A. (2015). Doing surveillance studies in Latin America: The insecurity context. Surveillance and Society, 13(1), 78-90.

Caldera, E., \& Parraguez Kobek, L. (2016). Cyber Security and Habeas Data: The Latin American response to information security and data protection. OASIS, 0(24), 109-128. 
https://doi.org/http://dx.doi. org/10.18601/16577558.n24.07

Cox, J. (2016). Privacy Activists Launch Database to Track Global Sales of Surveillance Tech. VICE. Retrieved from https://motherboard. vice.com/en_us/article/qkjznm/ privacy-activists-launch-database-to-track-global-sales-of-surveillance-tech

Fidler, M. (2014). Anarchy or Regulation: Controlling the Global Trade in Zero-Day Vulnerabilities. Stanford University. Retrieved from http:// purl.stanford.edu/zs241cm7504

Gellman, B., \& Poitras, L. (2013, June 7). U.S., British intelligence mining data from nine U.S. Internet companies in broad secret program. The Washington Post. Retrieved from https://www.washingtonpost. com/investigations/us-intelligence-mining-data-from-nine-us-internet-companies-in-broad-secret-program/2013/06/06/3a0c$0 \mathrm{~d}$ a 8 - c e b f - 11 e 2 - 8845 d970ccb04497_story.html

Greenwald, G. (2014). No Place to Hide. Metropolitan Books.

Ilv. (2015). Hacking Team, Chile y Ecuador. Tor Project. Retrieved from https://web.archive.org/ web/20150924185925/https:// people.torproject.org/ ilv/ht_ chile_ecuador.html
Lee, M., Greenwald, G., \& Marquis-Boire, M. (2015). BEHIND THE CURTAIN: A Look at the Inner Workings of NSA's XKEYSCORE. Retrieved January 14, 2018, from https:// theintercept.com/2015/07/02/ look-under-hood-xkeyscore/

Maciel, M., Foditsch, N., Belli, L., \& CasteIlon, N. (n.d.). Cyber security, privacy and trust. Trends in Latin America, 7-12. Retrieved from https:// bibliotecadigital.fgv.br/dspace/ bitstream/handle/10438/16706/ Cyber-Security\%2C Privacy and Trust Trends in the Latin American and Caribbean region and the way forward.pdf

Marczak, B., Scott-Railton, J., Senft, A., Poetranto, I., \& McKune, S. (2015). PAY NO ATTENTION TO THE SERVER BEHINDTHE PROXY: Mapping FinFisher's Continuing Proliferation. Retrieved from https://citizenlab.ca/2015/10/mapping-finfishers-continuing-proliferation/

Marczak, W. R., Scott-Railton, J., Marquis-Boire, M., \& Paxson, V. (2014). When Governments Hack Opponents: A Look at Actors and Technology. Proceedings of the 23rd USENIX Security Symposium, $511-$ 525.

Pérez de Acha, G. (2016). Hacking Team Malware para la Vigilancia en América Latina. Derechos Digitales. 
Scott-Railton, J., \& Marczak, B. (2016). THE MILLION DOLLAR DISSIDENT: NSO Group's iPhone Zero-Days used against a UAE Human Rights Defender. Retrieved January 14, 2018, from https://citizenlab. ca/2016/08/million-dollar-dissident-iphone-zero-day-nsogroup-uae/

Scott-Railton, J., Marquis-Boire, M., Guarnieri, C., \& Marschalek, M. (2015). PACKRAT: Seven Years of a South American Threat Actor. Retrieved January 14, 2018, from https:// citizenlab.ca/2015/12/packrat-report/

Setty, S. (2015). Surveillance, Secrecy, and the Search for Meaningful Accountability. Stanford Journal of International Law, 51(1), 69-103.

The ZMap Team. (2017). The Zmap Project. Retrieved from https://zmap. io/
Tinoco, T. (2015). Cyber ataque - parte 2. Quito, Ecuador: Ecuavisa. Retrieved from http://www.ecuavisa. com/video/video-vision-360/ destacados-360/cyber-ataque-parte-2-0

Wikileaks. (2011a). Strategic Telecommunication Network Monitoring. Retrieved October 29, 2017, from https://wikileaks.org/spyfiles/ docs/VASTECH_2011_StraTeleNetw_en.html

Wikileaks. (2011b). The Spy Files. Retrieved September 6, 2017, from https://wikileaks.org/the-spyfiles. html

Wikileaks. (2014). Spy Files. Retrieved August 28, 2017, from https://www. wikileaks.org/spyfiles/

Wikileaks. (2017). Vault 7: CIA Hacking Tools Revealed. Retrieved December 18, 2017, from https:// wikileaks.org/ciav7p1/ 
